\title{
Escala ECPP-FIM para evaluar las competencias profesionales psicomotrices en la formación inicial de maestros: evidencias de validez ECPP-FIM an assessment tool for evaluating the professional psychomotor skills within the initial teacher training: validity evidences
}

\author{
Lurdes Martínez-Mínguez, Laura Moya Prados, Laura Arnau-Sabatés
}

Universidad Autónoma de Barcelona (España)

\begin{abstract}
Resumen. Profesorado y estudiantado apuntan la necesidad de disponer de instrumentos de evaluación capaces de valorar el impacto del enfoque por competencias en la educación superior, y concretamente ante un incipiente perfil académico y profesional psicomotriz con competencias profesionales propias. El objetivo de este estudio ha sido obtener evidencias de validez y de fiabilidad para las puntuaciones de un instrumento para evaluar las competencias profesionales psicomotrices durante la formación inicial de maestros. Se aportan evidencias cualitativas basadas en el contenido y el proceso de respuesta del instrumento (ECPP-FIM), así como evidencias cuantitativas relacionadas con la estructura factorial y la fiabilidad. Para la validación cuantitativa, se aplicó el instrumento a una muestra de 318 estudiantes de maestro de educación infantil de cuatro universidades. Los resultados del análisis de validez de la estructura interna arrojaron una distribución en cinco factores interpretables capaces de explicar conjuntamente el 67,4\% de la varianza total que son: diseñar las sesiones de psicomotricidad, aplicar el sistema de actitudes del adulto, observar y evaluar el niño/a y la sesión, analizar y reflexionar la propia práctica y la de los otros, y relacionarse con la comunidad educativa y científica. Respecto al análisis de fiabilidad, el instrumento presenta coeficientes elevados de consistencia interna por escalas y evidencias de estabilidad temporal. En relación a las evidencias cualitativas, tanto especialistas como estudiantes, destacaron su relevancia y utilidad. Se concluye que el instrumento presenta evidencias de calidad psicométrica para ser utilizado en el contexto de la formación inicial del profesorado.
\end{abstract}

Palabras clave: instrumento de medida; enseñanza superior; formación de docentes; competencias psicomotrices; validez.

\begin{abstract}
Teachers and students point to the need to have assessment tools capable of assessing the impact of the competencybased approach in higher education, and specifically in the face of an incipient academic and psychomotor professional profile with their own professional competencies. The study seeks to obtain evidences of validity and reliability for the scores of an instrument to assess the professional psychomotor skills in the context of the initial teacher training. Qualitative evidence based on the content and the response process of the instrument (ECCP-FIM) are provided as well as quantitative evidence related to the factorial structure and reliability. For the quantitative validation, the instrument was applied to a sample of 318 students of initial teacher training from 4 universities. The analysis of the internal structure revealed the presence of a simple five-factor solution explaining the $67.4 \%$ of the variance and with a very good fit to the theoretical structure: designing the psychomotor sessions, applying the attitude system of the adults, observing and evaluation the children and the session, analysing and reflecting on their own practice, and being in relation with the educational and scientific community. With regards to reliability, the tool has a high internal consistency among the scales and presents evidences regarding temporal stability. Concerning the qualitative evidence, both specialists and students highlighted its relevance and utility. It is concluded that the instrument presents adequate psychometric guarantees to be used during the initial training of future teachers.
\end{abstract}

Keywords: measuring instruments; higher education; teacher education; psychomotor skills; validity.

\section{Introducción}

Las competencias son un campo de estudio complejo, multidisciplinar y poliédrico (Fernández \& Malvar, 2020). Afrontar unas demandas laborales cambiantes a mucha velocidad, es uno de los grandes retos de la sociedad actual y de los planes de estudio de las universi-

Fecha recepción: 01-04-21. Fecha de aceptación: 26-07-21

Lurdes Martínez-Mínguez

Lurdes.Martinez@uab.cat dades. Martínez Clares et al. (2019) apuntan que es necesario crear más puentes entre la universidad y el mercado laboral. La Educación Superior se estructura a partir de competencias entendidas como una combinación dinámica de conocimientos, habilidades, actitudes y destrezas que un profesional debe mostrar en su desempeño en cierta área ocupacional (Tuning, 2007).

Para Ferrada (2019) hay dos corrientes respecto a la sintonización de la «arquitectura curricular» del modelo competencial. Una más teórica-funcionalista con una construcción curricular de secuencia detallada y 
compartimentada de cada competencia. Y otra más socioconstructivista, con competencias personales situadas en el contexto en que se desempeñará el futuro profesional. Pero ninguna de las dos corrientes parece tener los efectos esperados. Según López-López et al. (2018) y Solà (2020), el profesorado universitario muestra mayoritariamente una percepción pesimista respecto al impacto del enfoque por competencias en la mejora de su práctica educativa y los esfuerzos desplegados no están favoreciendo los cambios estructurales esperados. Además, López-Pastor et al. (2019) aportan que profesorado en ejercicio considera que no siempre las competencias adquiridas o lo realizado durante la formación inicial, es transferible después en las escuelas. Por lo que se considera necesario seguir investigando y buscar instrumentos que muestren si es posible un enfoque competencial exitoso.

Son bastantes las propuestas que tipifican competencias (Bunk, 1994; Cano, 2005, 2007; Durán et al., 2009; Gallego, 2000; Mir \& Ferrer, 2014; Tobón, 2006). En Educación Superior, la clasificación más común las divide en genéricas o transversales y específicas, disciplinares o profesionales (Agencia Nacional Evaluación de la Calidad y Acreditación, 2005; Tuning, 2007). Para Tejada \& Ruiz (2016), las competencias profesionales guardan relación con lo que implica el ejercicio profesional; son definibles en la acción; son un proceso más que un estado; y están contaminadas de ética con uno mismo, con los demás y con la comunidad. Algunos autores consideran que el desarrollo de competencias profesionales no solo debe basarse en la acción, sino también relacionarse con situaciones reales de trabajo para que el desarrollo de la competencia sea realmente significativo (Del Pozo, 2013; González-Rivas et al., 2021; Rodríguez Aceituno \& Hernández-Pina, 2017). Una construcción significativa y el desarrollo de competencias profesionales, necesita un aprendizaje más responsable, autónomo, cooperativo y reflexivo (Biggs, 2010; Domingo \& Gómez, 2014), con instrumentos de evaluación capaces de valorarlo (Zubillaga-Olague \& Cañadas, 2021).

El interés por la psicomotricidad en los últimos tiempos ha ido creciendo progresivamente, conjuntamente con las competencias de esta disciplina que deben adquirir los profesionales de la educación infantil durante su formación inicial y permanente (Martínez-Mínguez, 2017) y los maestros especialistas o de la mención en educación física. Una de las causas son resultados de estudios sobre neurociencias que apuntan la importancia en los primeros años de vida donde los niños aprenden principalmente desde y con su cuerpo (Bueno, 2017). La especificidad de la disciplina psicomotriz hace necesario investigar sobre un perfil profesional con competencias propias (Martínez-Mínguez et al., 2017). Tuning (2007) ya apuntaba que la definición de perfiles académicos y profesionales y el desarrollo de los campos de las competencias que requieren las titulaciones, está íntimamente ligada a la identificación y desarrollo de las competencias y la manera de obtenerlas por medio de los diferentes currículos.

En la Orden ECI/3960/2007, de 19 de diciembre por la que se establece el currículo y se regula la ordenación de la educación infantil, se expone que el maestro de educación infantil puede y debe responsabilizarse de la docencia de las tres áreas del currículum. Pero muchas escuelas no fomentan suficientemente prácticas educativas que tengan en cuenta el área I (Conocimiento de uno mismo y autonomía personal) a través de la corporalidad de los niños y niñas en cualquier situación educativa (Cortés \& Moya, 2018; Moya, 2019), ni desde la cotidianidad ni desde sesiones específicas de psicomotricidad. Algo está pasando en las escuelas sobre quién, cómo y con qué formación o competencias debe ocuparse de esta área del currículum (Prieto \& Cerro, 2020), y en qué espacios debe desarrollarse (Prieto et al., 2020). En nuestro entorno se adoptan distintas modalidades debido a que no todos los profesionales después de su formación inicial se sienten suficientemente preparados para realizar la intervención psicomotriz en las escuelas. A veces se pide al maestro especialista o con mención de educación física que imparta la psicomotricidad en los cursos de educación infantil, cuando ni han sido formados específicamente ni se reconoce en las plantillas de las escuelas que deban impartir docencia antes de los 6 años. Otras veces se encarga a algún auxiliar o a algún generalista de educación infantil que no tenga tutoría, para que desarrolle esta disciplina en párvulos. Otra posibilidad es que exista algún maestro con formación específica a través de un postgrado o máster y se le asigne, pudiendo estar acompañado o no por el tutor/a. Pero hay pocos centros en los que sea el tutor/a quien imparta esta disciplina con su grupo-clase cuando es esta la modalidad por la que apuesta nuestro sistema educativo.

Teniendo en cuenta todas estas casuísticas, se precisa investigar si la formación inicial de los maestros de educación infantil o de educación física, ofrece las acciones formativas y evaluativas necesarias para que los futuros docentes puedan adquirir y desarrollar competencias profesionales psicomotrices y aplicarlas desde el inicio 
de su desarrollo profesional. Viscarro et al. (2012), ya apuntaban que era necesario revisar las funciones que debe desarrollar un maestro de educación infantil para aportar claridad a las competencias profesionales psicomotrices de los diplomados y graduados. Así que, por un lado, es necesario que los maestros durante su formación inicial desarrollen competencias sobre los fundamentos y objetivos de la psicomotricidad, su metodología y evaluación, el papel del adulto en este tipo de intervenciones, cómo difundir los resultados que provoca, etc. (Martínez-Mínguez \& Moya, 2017).Y por otro lado la educación superior debe contar con instrumentos de evaluación capaces de valorar los resultados de aprendizaje (Calatayud, 2021) de las competencias profesionales psicomotrices adquiridas.

La evaluación de competencias resulta imprescindible, pero sigue siendo una asignatura pendiente en el sistema universitario español (Pozo \& Bretones, 2015; Tejada \& Ruiz, 2016). La complejidad y características de las competencias profesionales exigen el empleo de instrumentos variados y la presencia de diferentes agentes evaluadores para poder llevar a cabo procesos de autoevaluación, heteroevaluación y coevaluación (Tejada \& Ruiz, 2016).

Realmente son escasas las aportaciones sobre competencias profesionales específicamente psicomotrices y su evaluación. En relación al perfil profesional de los psicomotricistas, pocos autores han realizado aportaciones para trazar algunas especificaciones relativas a las competencias profesionales del psicomotricista (Camps \& Mila, 2011; Martínez-Mínguez et al., 2018; Mila, 2018; Sánchez \& Llorca, 2008). Lo más desarrollado y considerado como marco de referencia encontrado es el Instrumento de Evaluación de las Competencias del Psicomotricista en el Ámbito Educativo (IECPAE) (Forcadell, 2014) centrado en la evaluación de las competencias profesionales del psicomotricista en su formación específica de postgrado o máster. En este instrumento se concretan siete competencias referidas a: programación, dispositivo de la sesión, clima, acompañamiento en el juego, observación, autorreflexión y relación con la comunidad.

Los planteamientos expuestos apuntan la necesidad de disponer de instrumentos de evaluación capaces de valorar el impacto del enfoque por competencias en general en la educación superior, y concretamente ante un incipiente perfil académico y profesional psicomotriz con competencias profesionales propias, durante la formación inicial del profesorado.

El objetivo principal de este estudio consiste en ob- tener evidencias de validez y fiabilidad para las puntuaciones de un instrumento para evaluar las competencias profesionales psicomotrices de los futuros maestros.

\section{Material y método}

Para elaborar la Escala de Competencias Profesionales Psicomotrices en la Formación Inicial de Maestros (ECPP-FIM), se han seguido diferentes fases a nivel metodológico que han permitido aportar evidencias cualitativas y cuantitativas relacionadas con el proceso de validación psicométrica del instrumento (ver Figura 1).

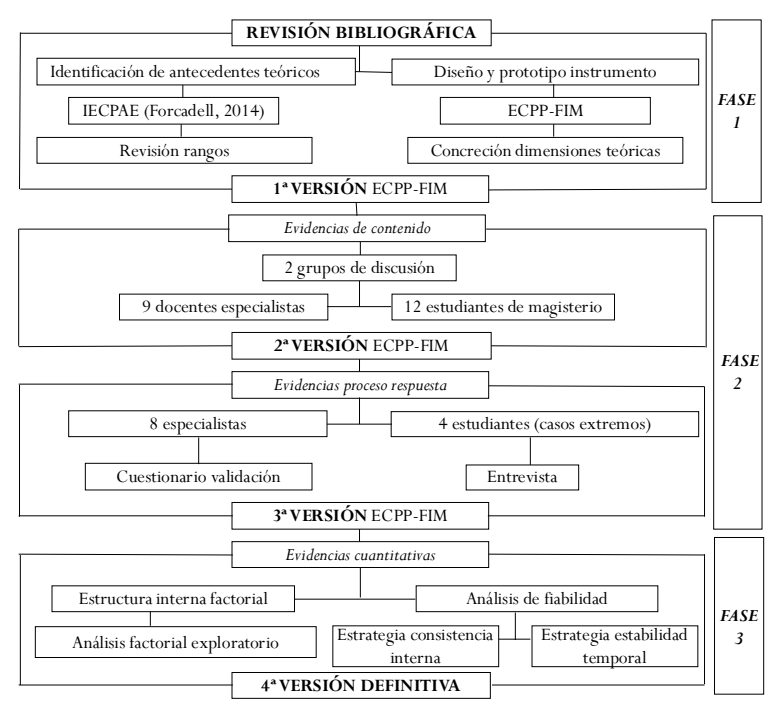

Figura 1. Diagrama del proceso de desarrollo del instrumento de evaluación de competencias profesionales psicomotrices en la formación inicial de maestros.

\section{Participantes}

Con el objetivo de obtener evidencias basadas en el contenido del instrumento se solicitó, a partir de un muestro intencional, la participación de nueve especialistas en el ámbito de la psicomotricidad y de la formación de maestros. Por otra parte, 12 estudiantes del Grado en Educación infantil de la Universitat Autònoma de Barcelona (UAB) también participaron en la validación del contenido de la escala. En un segundo momento, cuatro de estos 12 estudiantes fueron seleccionados, a partir de un muestreo por casos extremos (los dos con las puntuaciones más elevadas y los dos con puntaciones más bajas) para contestar una entrevista y obtener evidencias relacionadas con el proceso de respuesta. Asimismo, otros ocho especialistas académicos y profesionales en el ámbito de la psicomotricidad, valoraron la adecuación de la formulación de los ítems y otros aspectos relacionados con el proceso de respuesta (ver Figura 1).

Con el objetivo de analizar la estructura factorial y la fiabilidad de la ECPP-FIM se aplicó el instrumento 
(tercera versión) a una muestra de conveniencia. Esta muestra se concretó en 318 estudiantes de formación inicial de graduados en educación infantil, con edades comprendidas entre 18 y 29 años $(M=21.63$, SD $=$ 1.9) y de los que el $97.2 \%$ eran mujeres. Pese a no tratarse de una muestra aleatoria, el porcentaje de mujeres es representativo del perfil del estudiantado de los Grados en Educación Infantil (AQU, 2014). Los estudiantes provenían de las cuatro universidades participantes en el proyecto ARMIF (2017-0013): 146 de la Universitat Autònoma de Barcelona (UAB); 58 de la Universitat de Girona (UdG); 55 de la Universitat de Vic-Central de Catalunya (UVic); y 59 del campus de Teruel de la Universidad de Zaragoza (IniZar). Para comprobar la estabilidad temporal a partir del diseño test-retest, se seleccionó una submuestra de 32 estudiantes de cuarto curso de los 146 participantes de la $\mathrm{UAB}$ a la que se le administró el instrumento dos veces temporalmente diferenciadas.

Procedimiento y plan para el análisis de datos

Para analizar la estructura interna, se administró el instrumento durante el curso 2018-2019, en el contexto de las asignaturas que se imparten en el grado de Educación Infantil en las 4 universidades antes mencionadas: Educación Psicomotriz en los centros de Educación Infantil (cuarto curso UAB), Expresión Corporal, Psicomotriz y Aprendizaje (segundo curso UdG), Desarrollo Psicomotor y Expresión Corporal (segundo curso UVic) y Educación Física en Educación Infantil (tercer curso UniZar). Se trata de asignaturas en la que se trabajan temas relacionados con la psicomotricidad en niños de 0 a 6 años. Se aplicó el instrumento en un momento inicial, antes de empezar los contenidos de la asignatura.

Si bien el instrumento fue administrado por cinco profesores diferentes en sus respectivas asignaturas (uno o dos de cada universidad participante), se siguió un protocolo elaborado por las investigadoras principales para asegurar la homogeneidad en las condiciones de aplicación. Las consignas giraron principalmente en relación a textos consensuados sobre: la presentación de la escala de autoevaluación (objetivo general y específico del proyecto de investigación vinculado), las normas de aplicación (leer atentamente, contestar con sinceridad, temporalidad, interpretación gradientes y posibilidad comentarios) y un agradecimiento final (sobre el tiempo, la implicación y comunicar que su colaboración era imprescindible para investigar, mejorar e innovar).

Para analizar la estructura interna del ECPP-FIM y teniendo en cuenta la naturaleza exploratoria del estudio, se utilizó el análisis factorial exploratorio (AFE) (Lloret-Segura et al., 2014) mediante la técnica de extracción de ejes principales y rotación variamax, omitiendo las cargas inferiores al valor .40. Se contrastó el test de esfericidad de Bartlett (1950) como una prueba de seguridad y una condición necesaria para llevar a cabo el AFE.

Para analizar la fiabilidad se utilizaron dos estrategias. La primera fue comprobar el Coeficiente alfa de Cronbach para cada uno de los factores extraídos. Se consideraron adecuados valores mayores de .7, buenos los valores de .80 o mayores y muy buenos o excelentes los valores de .9 o mayores (Nullaly, 1978). Para la segunda estrategia, basada en la estabilidad temporal (diseño test-retest), se administró a 32 casos, provenientes de la muestra de la UAB, la misma escala en dos momentos cercanos en el tiempo. Para analizar la asociación entre la primera y la segunda pasación se utilizó el coeficiente de correlación de Pearson, aplicándose complementariamente la Rho de Spearman como medida de comprobación. En este caso, se siguieron las recomendaciones de Landis \& Koch (1977) y se consideró acuerdo bajo el rango de .21 a .40, acuerdo moderado de .41 a .60 , acuerdo bueno el rango de .61 a .80 y acuerdo excelente valores mayores a .81 .

\section{Resultados}

\section{Evidencias cualitativas: Contenido y Proceso de respuesta}

En el proceso de construcción del instrumento se desarrollaron diversas versiones modificadas a partir de los sucesivos estudios de validez (ver Figura 1). Estas fases permitieron ir depurando y optimizando la versión final del instrumento.

\section{Evidencias basadas en el contenido}

En la primera fase, se revisó la literatura existente sobre instrumentos de medida para evaluar las competencias psicomotrices de los estudiantes de magisterio. Se constató la escasez de instrumentos de estas características en el contexto de la formación inicial del profesorado. Sólo se identificó un instrumento validado, el IECPAE (Forcadell, 2014), compuesto por siete rangos y 73 ítems, creado con el objetivo de evaluar las competencias del psicomotricista pero aplicado a la formación de tercer ciclo (máster).

Con el objetivo de poder diseñar un instrumento que pudiera ser aplicado a la formación inicial de maes- 
tros, se creó la Escala de Competencias Profesionales Psicomotrices en la Formación Inicial de Maestros (ECPP-FIM). Para ello, se identificaron a nivel teórico, las dimensiones e indicadores de las competencias de psicomotricidad que deberían desarrollar los maestros durante la formación inicial (Camps \& Mila, 2011; Mas \& Anton, 2017; Rota, 2015; Sánchez \& Llorca, 2008) y se adaptaron algunos rangos e ítems inspirados en el IECPAE (Forcadell, 2014).

En la Tabla 1 se muestran las equivalencias iniciales entre los rangos definidos en el IECPAE (Forcadell, 2014) y las dimensiones propuestas en el ECPP-FIM.

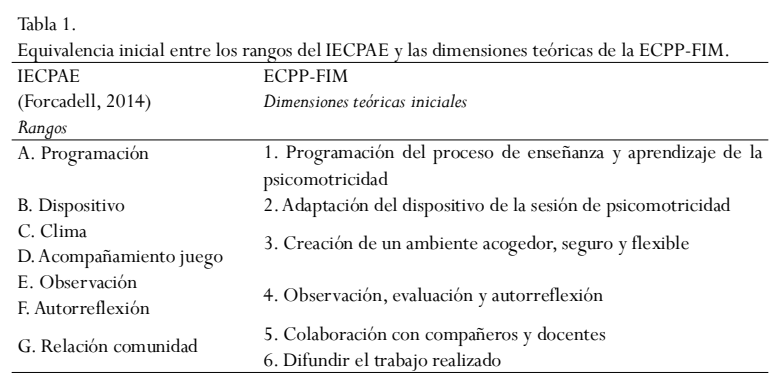

En la propuesta inicial del ECPP-FIM se definieron seis dimensiones y 29 ítems o elementos de competencia asociados a ellas. El aspecto más novedoso respecto al IECPAE (Forcadell, 2014) fue la incorporación de ítems que incluían elementos relacionados con el compartir y difundir el trabajo realizado. También se definió la longitud de la escala de medida optando, inicialmente, por una escala de respuesta de cinco grados. El redactado de los elementos de competencia se contextualizó y asimiló a aquello que acontece en el escenario natural del especialista en psicomotricidad para poder gozar de mayor validez ecológica.

Esta propuesta inicial (ver Figura 1) fue validada a partir de dos grupos de discusión. Uno formado por nueve docentes (dos profesoras universidad y siete maestros-psicomotricistas de las escuelas participantes en el proyecto) que destacaron la relevancia y representatividad de las seis dimensiones identificadas, con un alto grado de acuerdo. El otro grupo estaba formado por 12 estudiantes de educación infantil, y validaron, como especialistas en formación, aspectos relacionados con la utilidad y la comprensión de los ítems. A partir de sus valoraciones se refinó el redactado de algunos ítems para acabar de ajustar el contenido a las dimensiones propuestas, dando lugar a la segunda versión del instrumento.

Evidencias basadas en el proceso de respuesta

La segunda versión del instrumento (ver Figura 1) se sometió a una validación para controlar algunos factores que podrían afectar directamente la respuesta. Para ello, se contactó con otros ocho especialistas que validaron, a través de un cuestionario de validación, la univocidad (si/no) y la pertinencia de los elementos de cada subescala (de uno a cinco donde uno era nada pertinente y cinco muy pertinente) así como el formato, la claridad del redactado y comprensión de todos los ítems.

También se entrevistó a cuatro de los 12 estudiantes que habían contestado previamente el ECPP-FIM en la primera validación (los dos con puntuaciones más bajas y los dos con puntuaciones más altas) para preguntarles qué hicieron para contestar, cómo abordaron cada uno de los ítems, si les resultaron claros y comprensibles, si la longitud de la escala era adecuada, si consideraban que sobraba o faltaba algún ítem y si se veían reflejados en las puntuaciones. En general, los estudiantes encontraron el test útil para la autorregulación de su aprendizaje competencial, y dijeron verse representados en las puntuaciones de la escala.

Los principales cambios que se introdujeron se concretaron, fundamentalmente, en una mayor precisión lingüística y adecuación del vocabulario, así como en la eliminación, incorporación y/o reorganización de algunos ítems, concretándose la nueva versión en 35 ítems.

En esta segunda revisión se valoró la posibilidad de ajustar las dimensiones teóricas de la escala a cinco y no seis puesto que dos de las competencias «preparar el escenario de la sesión de psicomotricidad» y «Diseñar las sesiones de psicomotricidad» compartían indicadores muy relacionados con el diseño y la preparación de las sesiones. Este hecho implicó pasar de las seis dimensiones teóricas inicialmente propuestas en el ECPP-FIM a cinco dimensiones o competencias con mayor exclusividad y exhaustividad.

Además, siguiendo las recomendaciones de los expertos, se optó por una escala del uno al seis, asumiendo que el análisis factorial es más eficaz con escalas tipo Likert de cinco o más categorías (Frias-Navarro \& Pascual Soler, 2012) y que una escala par permite una mayor discriminación y polarización las respuestas (Simms et al., 2019).

A partir de los cambios realizados en la fase dos se generó la tercera versión del instrumento dando lugar a la tercera fase (ver Figura 1). La tercera versión del ECPP-FIM se aplicó a 318 estudiantes de cuatro universidades para poder obtener evidencias de validez cuantitativa relacionadas con la estructura interna y la fiabilidad de las puntuaciones del instrumento. 


\section{Evidencias cuantitativas: Estructura interna y fiabilidad}

A continuación, se presentan los resultados basados en la estructura interna del cuestionario. Para ello se ha realizado un AFE con componentes principales y la fiabilidad de las puntuaciones del instrumento a partir de las estrategias de consistencia interna y de estabilidad temporal (diseño test-retest).

\section{Evidencias basadas en la estructura interna}

Para explorar la estructura interna del ECPP-FIM se aplicó el AFE. El primer paso fue comprobar la media de adecuación muestral a través del coeficiente KMO ( $p=.937)$ y la prueba de esfericidad de Barlett (chicuadrado $=7871.7 ; p<.001)$, ambas permitieron concluir que el AFE resultaba pertinente. Las comunalidades de los ítems oscilaban entre .453 y .816 .

Después de realizar la rotación de los datos y de revisar diferentes soluciones se optó por una estructura de cinco factores por ser la que mejor encajaba con los componentes teóricos.

En este sentido, los 35 ítems del instrumento se distribuyeron en cinco factores interpretables, capaces de explicar conjuntamente el $67.4 \%$ de la varianza total.

El primer factor: Diseñar las sesiones de psicomotricidad, explica una varianza del $43.1 \%$ y agrupa 12 ítems relacionados con los conocimientos necesarios para poder diseñar y preparar las sesiones y el escenario de la clase. Por ejemplo: «Definir los objetivos a alcanzar durante la intervención psicomotriz».

El segundo factor: Aplicar el sistema de actitudes del maestro / a-psicomotricista, explica una varianza del $8.8 \%$ y agrupa 10 ítems relacionados con el rol docente en la intervención psicomotriz durante la sesión. Por ejemplo: «Reconocer y respetar al niño/a desde su propia individualidad».

El tercer factor: Relacionarse con la comunidad educativa y científica, explica una varianza del 6.9\% y agrupa cinco ítems sobre la capacidad del docente para trabajar en equipo, documentar y difundir su trabajo. Por ejemplo: «Mantener una buena comunicación y relación con las familias de los niños/as».

El cuarto factor: Observar y evaluar el niño/a y la sesión de psicomotricidad, explica una varianza del 5.1\% y agrupa tres ítems relacionados con la elaboración y utilización de instrumentos de observación y evaluación tanto de los niños/as como de la sesión de psicomotricidad. Por ejemplo: «Ubicarse en la sala para ir favoreciendo la observación de los niños y niñas».

Y, finalmente, el quinto factor: Analizar y reflexionar la propia práctica y la de los demás, explica una varianza del $3.4 \%$ y agrupa tres ítems relacionados con la capacidad de reflexionar de forma crítica sobre la propia práctica (autoevaluación) y la de los demás (co-evaluación). Por ejemplo: «Ser capaz de valorar de forma constructiva la actuación de otros compañeros/as y/o profesionales (coevaluación)».

La estructura extraída tiene una alta correspondencia con las dimensiones postuladas teóricamente, lo que supone una constatación empírica de las mismas.

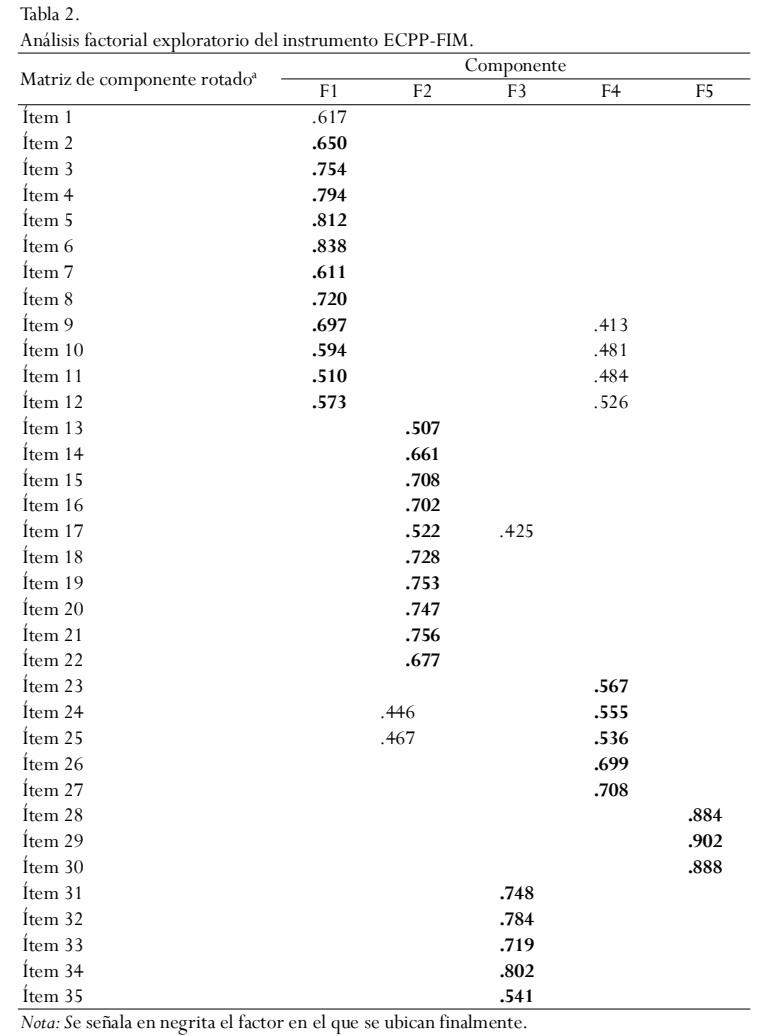

La matriz de cargas factoriales (Tabla 2) muestra que todos los ítems del instrumento saturaban en uno de los cinco factores. Sólo seis ítems saturaron simultáneamente y alto en dos factores: los ítems 10, 11 y 12 (factores uno y cuatro), el ítem 17 (factores dos y tres) y el 24 y 25 (factores dos y cuatro). Se consideró que lo más pertinente era que cada ítem permaneciera en el factor en el que tenía la saturación más alta. En cuatro casos (ítems 10, 11, 12 y 17), se decidió reformular el redactado para hacerlo más claro, unívoco y reducir así posibles ambigüedades en la versión final del instrumento.

Ítem 10. Organizar la distribución del espacio en función de los objetivos y las características de los niños/as de cero a ocho años. Se decidió substituir el redactado por: «Planear la distribución del espacio de la sala de psicomotricidad».

- Ítem 11. Adaptar la duración de las diferentes 
fases de la sesión en función de los objetivos y de las características de los niños/as de cero a ocho años. Se decidió substituir el redactado por: «Programar la duración de las diferentes fases de la sesión».

Ítem 12. Escoger el tipo, cantidad y distribución del material, en función de los objetivos y las características de los niños/as. Se decidió substituir el redactado por: «Concretar el material, cantidad y su distribución en la sala de psicomotricidad».

Ítem 17. Mantener una actitud firme ante los incumplimientos de las normas de la sesión. Se decidió substituir el redactado cambiando la palabra «firme» por «coherente».

En el caso de los ítems 23 y 24, se eliminaron y excluyeron de la interpretación ya que ambos estaban incluidos conceptualmente en los ítems 26 y 27.

\section{Evidencias de fiabilidad}

Los coeficientes alfa de Cronbach de cada subescala se presentan en la Tabla 3. Se observa que la escala presenta un coeficiente de consistencia interna entre buena y excelente por factores (coeficientes alfa entre $.874 \mathrm{y}$ .942).

Tabla 3.

Análisis de la fiabilidad de alfa de Cronbach por factores.

\begin{tabular}{lcc}
\hline Factores & $\mathrm{N}^{\circ}$ ítems & alfa \\
\hline Factor 1. Diseñar las sesiones de psicomotricidad & 12 & 942
\end{tabular} Fer 10 - De- 925 . Factor 4. Observar y evaluar el niño/a y la sesión de psicomotricidad Factor 5. Analizar y reflexionar la propia práctica y la de los demás

En la Tabla 4 se muestran los coeficientes de correlación test-retest para cada subescala o competencia evaluada en la escala ECPP-FIM:

Tabla 4

Coeficientes de correlación test-retest y niveles de significación.

Competencia evaluada en el Test-Retest del ECPP- Coeficiente de correlación $r \quad$ Nivel de FIM

entre la primera y segunda significación Factor 1. Diseñar las sesiones de psicomotricidad Factor 2. Aplicar el sistema de actitudes del maestro/a-psicomotricista

Factor 3. Relacionarse con la comunidad educativa y científica

Factor 4. Observar y evaluar el niño/a y la sesión de psicomotricidad

Factor 5 . Analizar y reflexionar la propia práctica y la de los demás evaluación .369 significacion $<.05$

\section{.501} $<.005$

Nota: $\mathrm{ns}=$ no significativo

Los coeficientes de correlación test-retest han resultado bajos para cada una de las subescalas, a excepción de la escala tres «Relacionarse con la comunidad educativa y cientifica» que presenta un coeficiente moderado $(\mathrm{r}=.501)$. Las comprobaciones no paramétricas con la Rho de Spearman corroboraron los resultados obtenidos. El hecho de que los coeficientes de estabilidad temporal sean, en general, bajos, era en parte esperable, y puede atribuirse a diversos factores relacionados con el efecto aprendizaje y con la intervención que se realizó en el aula para mejorar el desarrollo competencial del estudiantado en el marco de la asignatura «Educación Psicomotriz en los centros de Educación Infantil» en la UAB. Los elementos de competencia incluidos en el factor tres son, por su contenido, los menos trabajados en el contexto de la asignatura y seguramente esto explicaría una mayor estabilidad en las puntuaciones de esta escala. Contrariamente, los elementos de competencia asociados al resto de escalas (factor uno, dos, cuatro y cinco) se incorporaron más formalmente en los contenidos curriculares y se realizó una intervención específica para mejorarlos. En este sentido, se esperaba que estas puntuaciones asociadas a estas escalas se modificaran y no se mantuvieran estables.

\section{Discusión y conclusiones}

Se han realizado distintos análisis para obtener información en relación a las propiedades psicométricas de las puntuaciones del instrumento ECPP-FIM. Esta evidencia preliminar indica que se está avanzando en la dirección esperada. La información obtenida indica la adecuación de los ítems y las subescalas desarrolladas y la utilidad del instrumento aportado.

Desde un punto de vista de las evidencias basadas en la estructura interna, el análisis AFE sugiere la existencia de cinco factores, relevantes e interpretables, que explican el $67.4 \%$ de la varianza y que se corresponden con las dimensiones de las competencias profesionales psicomotrices postuladas teóricamente (Camps \& Mila, 2011; Martínez-Mínguez et al., 2018; Mas \& Anton, 2017; Mila 2018; Rota, 2015; Sánchez \& Llorca, 2008). En este sentido, el análisis factorial ha permitido corroborar la estructura de las definiciones de las competencias o subescalas así como la agrupación de los elementos asociados a cada una de las competencias. Una vez concluido el AFE, se decidió incorporar un ítem en el primer factor al ver que no quedaba suficientemente recogida la importancia del juego en psicomotricidad: «Conocer la evolución del juego psicomotor en niños de cero a ocho años». Asimismo, también se incorporó un ítem relacionado con la adaptación de las fases en el factor de la actitud del adulto en el desarrollo de las sesiones: «Adecuar la duración de las diferentes fases de la sesión». Finalmente, después de añadir estos dos ítems, el ECPP-FIM se compone de cinco competencias y 35 ítems o elementos de competencia sobre los que inci- 
dir si se quieren mejorar las competencias profesionales psicomotrices en el contexto de la formación inicial de maestros (ver anexo). Sería necesario en futuras investigaciones, poder llevar a cabo un análisis factorial confirmatorio para ratificar su estructura interna, y corroborar la bondad de ajuste de la solución factorial aportada. Asimismo, también sería interesante hallar evidencias de relación de la escala con otras variables que contribuyan a reforzar su valor predictivo como podría ser relacionar las puntuaciones con otros criterios externos como por ejemplo las calificaciones o media del expediente académico.

Sobre la fiabilidad se puede considerar que la versión del instrumento presenta una muy buena consistencia interna de las puntuaciones de las cinco subescalas o dimensiones. No obstante, el análisis de estabilidad temporal muestra coeficientes menos satisfactorios, siendo este resultado esperable puesto que se realizó una intervención en el contexto de la asignatura para mejorar las competencias profesionales psicomotrices asociadas a la ECPP-FIM antes de la segunda aplicación. Es importante destacar que el factor menos trabajado en la intervención («Relacionarse con la comunidad educativa y científica») fue también el factor menos sensible al cambio, hecho que permitió constatar que los resultados del test retest iban en el sentido deseado.

Con las evidencias reunidas hasta el momento, el instrumento ECPP-FIM se configura como un buen candidato para preparar una intervención pedagógica que permita diagnosticar y mejorar el nivel de desarrollo de las competencias profesionales de psicomotricidad en el contexto de la educación infantil, a partir de procesos de autoevaluación, coevaluación o hetero-evaluación. Esta escala también puede contribuir a un mejor equilibrio entre la enseñanza que se ofrece en la educación superior y los resultados del aprendizaje competencial del egresado (Martínez Clares et al., 2019). Por otra parte, su carácter copyleft posibilita una amplia utilización por parte de docentes universitarios que imparten formación en psicomotricidad en los grados de educación infantil y primaria, de los propios estudiantes y también a maestros en ejercicio que quieran revisar y mejorar sus competencias profesionales de psicomotricidad. Deseamos que la ECPP-FIM sea un instrumento como las prácticas que apuntan Pérez-Pueyo \& Hortigüela-Alcalá (2019): efectivo, sostenible, capaz de mantenerse en el tiempo, con efectos duraderos, replicable y que demuestre impacto positivo y tangible de mejora.

El estudio presenta algunas limitaciones que en el futuro deberían abordarse. La administración de la escala fue realizada por diferentes profesores. Si bien se consensuó un protocolo de aplicación, las condiciones de aplicación se hubieran controlado mejor si hubiera sido la misma persona la que aplicara el instrumento en todos los grupos. Sería necesario incluir algún ítem de deseabilidad social que permitiera contrarrestar tal efecto, así como algún ítem de control de falsedad que permitiera comprobar y eliminar las respuestas contestadas azarosamente.

El instrumento aporta un resultado empírico favorable para la evaluación de las competencias psicomotrices en futuros maestros y su formato, aporta además validez ecológica al permitir contextualizar las respuestas a situaciones que acontecen en el escenario natural del especialista en psicomotricidad. En este sentido, la aplicación de este instrumento en el contexto de la formación inicial de magisterio, puede ayudar a compensar los resultados que detectaron MartínezMínguez \& Flores (2014) en su estudio, quienes comprobaron que en la formación inicial de maestros se desarrollan más capacidades cognitivas como memorizar y recordar, que las de aplicar o analizar en entornos profesionales auténticos. La escala ECPP-FIM posibilita iniciar la preparación de las competencias profesionales psicomotrices en la formación básica de maestro al tiempo que permite llevar a cabo una formación con carácter profesionalizador.

Otra posibilidad de aplicación la escala ECPP-FIM podría ser en el ejercicio profesional de los maestros en activo como herramienta de autoevaluación y autorreflexión crítica. Esta se apunta como una posible línea de investigación futura, juntamente con replicar los resultados en otras muestras y contextos formativos similares.

\section{Agradecimientos}

Este estudio se ha llevado a cabo dentro de un proyecto de investigación competitivo:

- Autoevaluación de competencias de los estudiantes y desarrollo profesional de los maestros. Mejora de la práctica e innovación docente trabajando por proyectos de aprendizaje psicomotores co-tutorados universidad-escuela. Convocatoria de Ayudas de investigación para la mejora de la Formación Inicial de Maestros (ARMIF), Resolución, EMC/ 2219/2017 de 14 de septiembre (DOGC núm. 7461 de 26 de septiembre de 2017) de la Agencia de Gestión de Ayudas Universitarias y de Investigación (AGAUR). Referencia: 2017-ARMIF-00013. 


\section{Referencias}

Agencia Nacional Evaluación de la Calidad y Acreditación. (2005). Libro blanco título de Grado en Magisterio, 1. Madrid: Sirius.

AQU (2014). Universitat i Treball a Catalunya 2014. Barcelona: Agencia para la Calidad del Sistema Universitario de Cata-

luña. Recuperado de https: / / www.aqu.cat/doc/ doc_14857668_1.pdf

Bartlett, M.S. (1950). Tests of significance in factor analysis. British Journal of Mathematical and Statistical Psychology, 3, 77-85.

Biggs, J. (2010). Calidad del aprendizaje universitario. (4 ${ }^{\mathrm{a}}$ edición). Madrid: Narcea.

Bueno, D. (2017). Neurociencia para educadores. Barcelona: Octaedro.

Bunk, G. P. (1994). La transmisión de las competencias en la formación y perfeccionamiento profesionales de la RFA. Formación Profesional. Revista Europea, 1/1994. 8-14. Recuperado de https://dialnet.unirioja.es/descarga/articulo/131116.pdfç

Calatayud Salom, M. (2021). Metáforas evaluativas del alumnado universitario de Educación Física. Necesidad de un cambio radical Retos, 41, 854-865. https: / / doi.org/ 10.47197/retos.v41i0.82706

Camps, C. \& Mila, J. (Coord.). (2011). El psicomotricista en su cuerpo. De lo sensoriomotor a la transformación psíquica. Buenos Aires, Argentina: Miño y Dávila.

Cano, E. (2005). Cómo mejorar las competencias de los docentes: guía para la autoevaluación y desarrollo de las competencias del profesorado. Barcelona: Graó.

Cano, E. (2007). Las competencias de los docentes. En Ministerio de Educación y Ciencia (Ed.), El desarrollo de competencias docentes en la formación del profesorado (pp. 33 60). Madrid: Secretaria GeneralTécnica.

Cortés, F. \& Moya, L. (2018). La psicomotricidad des de una mirada interdisciplinaria. Revista Infancia, 223, 9-12.

Costello, A.B. \& Osborne, J.W. (2005). Best practices in exploratory factor analysis: Four recommendations for getting the most from your analysis. Practical Assessment, Research \& Evaluation, 10(7), 1-9. Recuperado de https: / / s c h o l a r w orks.u mas s.ed u / c g i / viewcontent.cgi?article $=1156 \&$ context $=$ pare

Del Pozo, J.A. (2013). Competencias profesionales. Herramientas de evaluación: el portafolios, la rúbrica y las pruebas situacionales. Madrid: Narcea.

Domingo, A. \& Gómez, V. (2014). La práctica reflexiva. Bases, modelos e instrumentos. Madrid: Narcea.

Durán, J.A., Marcano, N. \& Moronta, M. (2009). Competencias investigativas del docente de educación básica. Revista Laurus, 15(39), 138-165. Recuperado de https:// www.redalyc.org/pdf/761/76120651007.pdf

Fernández, M. D. \& Malvar, M. L. (2020). Las competencias emocionales de los orientadores escolares desde el para- digma de la educación inclusiva. Revista de Investigación Educativa, 38(1), 239-257. Recuperado de https:// doi.org/10.6018/rie.369281

Ferrada, D. (2019). La sintonización en la formación inicial docente. Una mirada desde Chile. Revista Electrónica de Investigación Educativa, 21, e39, 1-14. Recuperado de https: / / doi.org/10.24320/redie.2019.21.e39.2113

Forcadell, X. (2014). La evaluación de las competencias del psicomotricista en el ámbito educativo para mejorar su desarrollo profesional. (Tesis doctoral, Universitat Autònoma de Barcelona). Recuperado de http://www.tdx.cat/ handle/10803/285371

Frías-Navarro, D., \& Pascual Soler, M. (2012). Exploratory factor Analysis (EFA) in consumer behavior and marketing research. Suma Psicológica, 19(1), 47-58.

Gallego, M. (2000). Gestión humana basada en competencias. Contribución efectiva al logro de los objetivos organizacionales. Revista Universidad EAFIT, 36(119), 6371. Recuperado de https://publicaciones.eafit.edu.co/ index.php/revista-universidad-eafit/article/view/1026 González-Rivas, R., Zueck Enríquez, M., Baena-Extremera, A., Marín Uribe, R., Soto Valenzuela, M., \& Irigoyen Gutiérrez, H. (2021). Desarrollo de competencias en educadores físicos en formación a través de la inclusión de programas de educación aventura en México. Retos, 42, $126-135$. https: / / doi.org/10.47197/ retos.v42i0.85840

Landis J.R. \& Koch G.G. (1977). The measurement of observer agreement for categorical data. Biometrics 33, 159-174.

López-López, M.C., León-Guerrero, M.J. \& Pérez-García, P. (2018). El enfoque por competencias en el contexto universitario español. La visión del profesorado. Revista de Investigación Educativa, 36(2), 529-545. https://doi.org/ 10.6018/rie.36.2.314351

López Pastor, V., Molina Soria, M., Pascual Arias, C., \& Manrique Arribas, J. (2019). La importancia de utilizar la Evaluación Formativa y Compartida en la Formación Inicial del Profesorado de Educación Física: los Proyectos de Aprendizaje Tutorado como ejemplo de buena práctica. Retos, 37, 620-627. https://doi.org/10.47197/ retos.v37i37.74193

Lloret-Segura, S., Ferreres-Traver, A., Hernández-Baeza, A. \& Tomás-Marco, I. (2014). El análisis factorial exploratorio de los ítems: una guía práctica, revisada y actualizada. Anales de Psicología, 30(3), 1151-1169. Recuperado de: https: / /www.redalyc.org/articulo.oa?id=16731690031

Martínez Clares, P., González Lorente, C. \& Rebollo Quintela, N. (2019). Competencias para la empleabilidad: un modelo de ecuaciones estructurales en la Facultad de Educación. Revista de Investigación Educativa, 37(1), 57-73. Recuperado de http://dx.doi.org/10.6018/ rie.37.1.343891

Martínez-Mínguez, L. (2017). La formación del 
psicomotricista a lo largo de la vida profesional. En M. Mas \& M. Anton (Coords.), Psicomotricidad educativa: avanzando paso a paso (pp. 51-72). Octaedro.

Martínez-Mínguez, L. \& Flores, G. (2014). Profesorado y egresados ante los sistemas de evaluación del alumnado en la formación inicial del maestro de educación infantil. RIDU. Revista Digital de Investigación en Docencia Universitaria, 8 (1), 29-50. http://revistas.upc.edu.pe/ index.php/docencia/article/view/371/344

Martínez-Mínguez, L. \& Moya, L. (2017). La opinión de los estudiantes: la tutoría académica en la adquisición de competencias profesionales de educación física en los graduados en Educación Infantil. Didacticae, 2, 71-88. http:// revistes.ub.edu/index.php/didacticae/article/view/ $20191 / 22350$

Martinez-Mínguez, L., Rota, J. \& Anton, M. (Coords.) (2017). Psicomoticitat, escola i currículum. Octaedro.

Martínez-Mínguez, L., Rota, J. \& Moya, L. (2018). Las competencias del trabajo corporal en la formación de psicomotricistas. En Federación de Asociaciones de Psicomotricidad del Estado Español, Actas del VI Congreso Estatal de Psicomotricidad. Psicomotricidad: Más vale prevenir. FAPee. (En prensa).

Mas, M. \& Anton, M. (Coord.). (2017). Psicomotricidad educativa: avanzando paso a paso. Barcelona: Octaedro.

Mila, J. (2018). Los estudios de psicomotricidad en la Universidad de la República de Uruguay. Percepción de las competencias sobre formación corporal de los estudiantes (Tesis Doctoral, Universidad de Murcia). Recuperado de https:/ / digitum.um.es/digitum/bitstream/10201/61659/1/ Tesis\%20MILA\%20DEMARCHI\%20.pdf

Mir, M.L. \& Ferrer, M. (2014). Aproximación a la situación actual de la formación del profesorado de educación infantil. Revista Electrónica Interuniversitaria de Formación del Profesorado, 17(2), 235-255. Recuperado de https:// doi.org/10.6018/reifop.17.2.181441

Moya, L. (2019). La corporeidad de los maestros y las maestras en su formación inicial. Una oportunidad para (re)conocerse y (re)descubrirse como docentes. Aula de Infantil, 98, 20-23.

Muñoz-San Roque, I., Martín-Alonso, J. F., Prieto-Navarro, L. \& Urosa-Sanz, B. (2016). Autopercepción del nivel de desarrollo de la competencia de aprender a aprender en el contexto universitario: propuesta de un instrumento de evaluación. Revista de Investigación Educativa, 34(2), 369383. Recuperado de http://dx.doi.org/10.6018/ rie.34.2.235881

Nunnally, J. C. (1978). Psychometric Theory (2nd ed.). New York: McGraw-Hill.

Orden ECI/3960/2007, de 19 de diciembre, por la que se establece el curriculum y se regula la ordenación de la educación infantil, Boletín Oficial del Estado, 5, 10161036 (2007). Recuperado de https://www.boe.es/ boe/dias/2008/01/05/pdfs/A01016-01036.pdf
Pérez-Pueyo, A., \& Hortigüela-Alcalá, D. (2019). ¿Y si toda la innovación no es positiva en Educación Física? Reflexiones y consideraciones practices. Retos, 37, 579-587. https://doi.org/10.47197/retos.v37i37.74176

Pozo, C. \& Bretones, B. (2015). Dificultades y retos en la implantación de los títulos de grado en las universidades españolas. Revista de Educación, 267, 147-172. Recuperado de http://www.educacionyfp.gob.es/dam/ jcr:986f863e-d400-4f79-a 5ad-317df49e934b/ re36706-pdf.pdf

Prieto Prieto, J., \& Cerro Herrero, D. (2020). Percepciones de futuros maestros sobre motricidad en educación infantil: un estudio exploratorio en estudiantes de último curso. Retos, 39, 155-162. https://doi.org/10.47197/ retos.v0i39.78618

Prieto Prieto, J., Galán Jiménez, N., Barrero Sanz, D. , \& Cerro Herrero, D. (2020). La sala de psicomotricidad para el trabajo de educación física en educación infantil: un estudio exploratorio. Retos, 39, 106-111. https://doi.org/ 10.47197/retos.v0i39.78398

RodriguezAceituno, P. \& Hernández Pina, F. (2017). Didáctica de la Motricidad en la formación de profesores de educación infantil. Retos, 34, 25-32. https://doi.org/ $10.47197 /$ retos.v0i34.58416

Rota, J. (2015). La intervención psicomotriz. De la práctica al concepto. Barcelona: Octaedro.

Sánchez, J. \& Llorca, M. (2008). El rol del psicomotricista. Revista Interuniversitaria de Formación del Profesorado, 62 (22,2), 35-60. Recuperado de https:// www.redalyc.org/pdf/274/27414780004.pdf

Solà Santesmases, J. (2020). Estudio de la transversalidad de los contenidos en Educación Física a través de los currículos competenciales. Retos, 40, 419-429. https: / / doi.org/10.47197/retos.v0i40.81783

Tejada, J. \& Ruiz, C. (2016). Evaluación de competencias profesionales en Educación Superior: Retos e implicaciones. Educación XX1, 19(1), 17-38. Recuperado de http:// dx.doi.org/10.5944/educxx1.12175

Tobón, S. (2006). Las competencias en la educación superior. Políticas de calidad. Bogotá, Colombia: ECOE.

Tuning. (2007). Competences. University of Groninge. University of Deusto. Recuperado de http:// www.unideusto.org/tuningeu/competences.html

Viscarro, I., Anton, M. \& Cañabate, D. (2012). Perfil y formación de los profesionales que realizan la práctica psicomotriz en la etapa de educación infantil. El caso de las comarcas de Tarragona. EDUCAR, 48(2), 321-344. Recuperado de http://www.redalyc.org/pdf/3421/ 342130839008.pdf

Zubillaga-Olague, M., \& Cañadas, L. (2021). Diseño y validación del cuestionario «\#EvalEF» para conocer el proceso de evaluación desarrollado por los docentes de educación física. Retos, 42, 47-55. https: / / doi.org/10.47197/ retos.v42i0.86627 


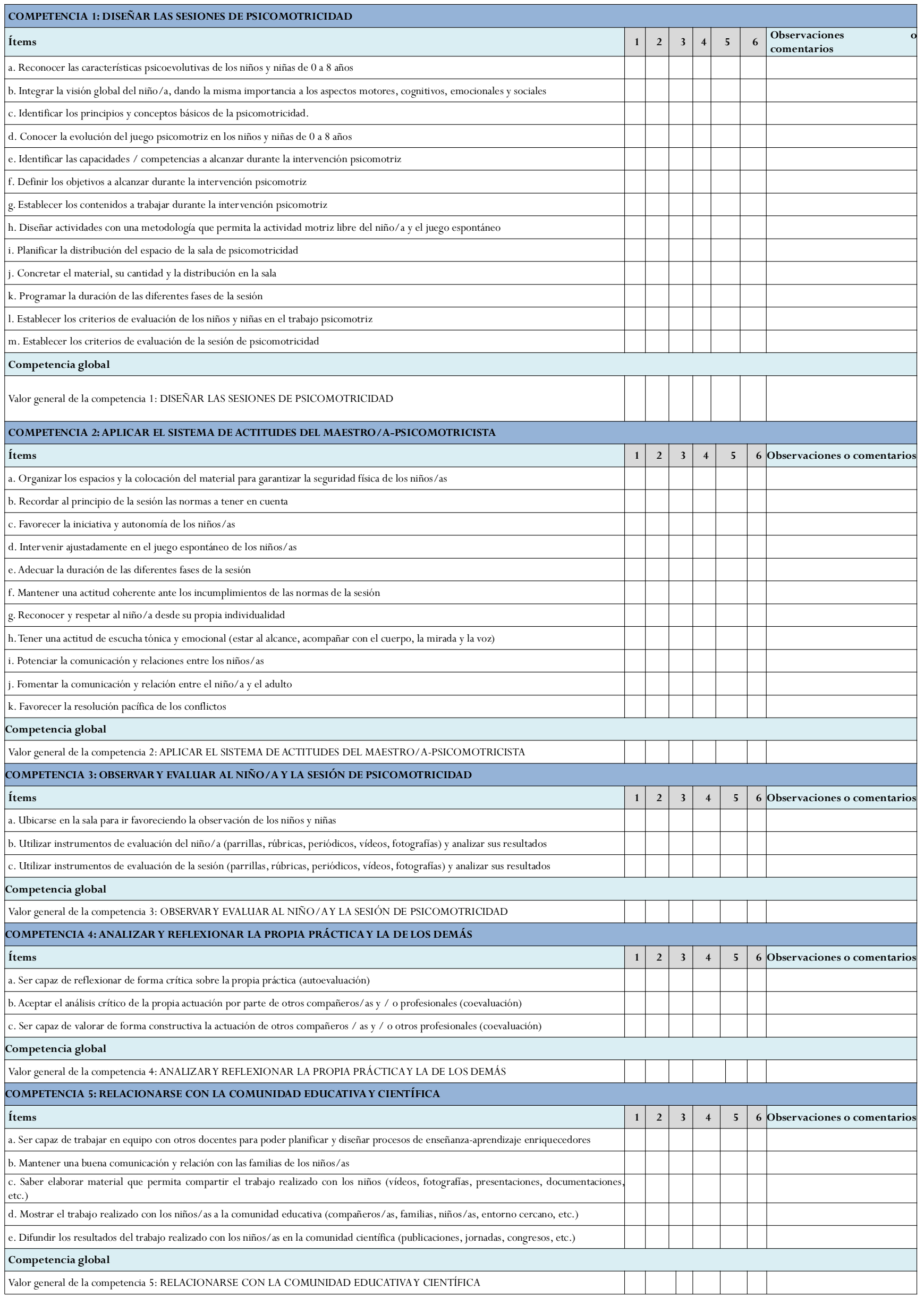

